\title{
An improved quantum genetic algorithm for the Resource Constrained Project Scheduling
}

\author{
Li Mingliang $^{1, a}$, Song Xueyan ${ }^{2, b}$ \\ School of Computer Science and Technology,Tianjin University, Tianjin 30072,China \\ Tianjin Key Laboratory of Cognitive Commputing and Application, Tianjin University,Tianjin \\ 30072,China \\ aemail: 295554150@qq.com
}

Keywords: Quantum computing; quantum genetic algorithm; resource-constrained; project scheduling

\begin{abstract}
In this paper, proposes an improved quantum genetic algorithm based on RCPSP. Project implementation process often involves the sharing of resources and conflicts between the different projects, so in the case of how resource-constrained project scheduling resources to meet the requirements of different projects and shorten the duration of each project is an important issue. Algorithm is based on the priority activities quantum encoding, combined with adjacency matrix storage activities, effectively solved the phenomenon of illegal activity scheduling, using preemptive mode of resource allocation to schedule resources, thus avoiding resource allocation conflicts problem. Experiments show that, through multiple iterations of the computer's parallel computing can be effectively come to meet the multi-project resource constrained scheduling.
\end{abstract}

\section{Introduction}

The first quantum algorithm to solve the decomposition of large number of factors and put forward in 1996 quantum algorithms for random database search have attracted much attention due to their unique computational performance, and have rapidly become a hotspot in the world. Quantum genetic algorithm is a probability optimization algorithm based on quantum computing principle. It has the advantages of small population size, fast convergence speed and strong global search ability. It is mainly applied to combinatorial optimization, such as Traveling Salesman problem, Knapsack problem. At present, all kinds of quantum genetic algorithms (such as mixed quantum, parallel quantum) are almost all varieties of the quantum genetic algorithm. According to the literature [1].These algorithms mainly have the following problems:

(1) By measuring the state of the quantum bits to obtain binary solution, which is a probabilistic operation process, with great randomness and blindness, in the process evolution of the population, some individuals will inevitably produce degradation.

(2) For the rotation angle of the quantum revolving door, it is almost based on the query table or its variants, which affects the efficiency of the algorithm because of the multi-path condition judgment. For the size of the rotation angle, the existing method treats all the population equally, without considering the differences between the chromosomes. Based on the problems above, this paper presents an improved quantum genetic algorithm.

\section{Problem Description}

A project contains $m+1$ activities, one of the inputs of an instance of RCPSP is a set of activities $\mathrm{j}=\left\{j_{0}, j_{1}, j_{2}, \ldots, j_{m+1}\right\}$, and the activities are independent besides the shared resources.. Activities $j_{0}$ and $j_{m+1}$ are dummy activities, serving as the only start and the end of the project. All virtual activities that do not consume resources and have an execution time of 0 , and represent the start and end of the entire project. Due to technical requirements, there is a close relationship among some activities, such as activity $j$ cannot start until any of its immediate activities $i, i \in p_{j}\left(p_{j}\right.$ is the immediate active 
set of activity $i$ ) has been done. The structure of the whole project is represented by a directed network diagram, in which the nodes represent the activities and the arcs represent the immediate relationship among the activities. Each activity sequence number in the figure should be ensured that the activity number in $p_{i}$ is less than $j$. A feasible scheduling scheme means that the start time of each activity has been determined, and the preconditions and resource constraints are satisfied. According to the literature [2]

The assumption of activity $j(j=0,1,2, \ldots, J)$ completed needs the $k . k$ is a set of renewable resource types; each type $k$ has a finite capacity $r_{j k}$ hat remains unchanged, Execution time is $d_{j}$. the starting time of activity $j$ is $S_{j}, A_{t}$ is the active set in $(t-1, t)$ time period, We can now provide a conceptual formulation of RCPSP:

$$
\begin{aligned}
& \quad \min S_{j} \\
& \text { s.t. } S_{j}-S_{i} \geq d_{i}, i \in P_{j} \\
& \sum_{j \in A_{t}} r_{j k} \leq R_{k}, t=1,2, \ldots, S_{J}, k=1,2, \ldots, K
\end{aligned}
$$

The constraint set (1) describes the precedence constraints between the activities. These are all of the finish-to-start (FS) type: the successor cannot be started before the predecessor is finished .Further in this text, we also use start-to-start (SS).(2) represents the resource constraints, A schedule s that respects constraints (1) -(2) is called a feasible schedule. According to the literature [3]

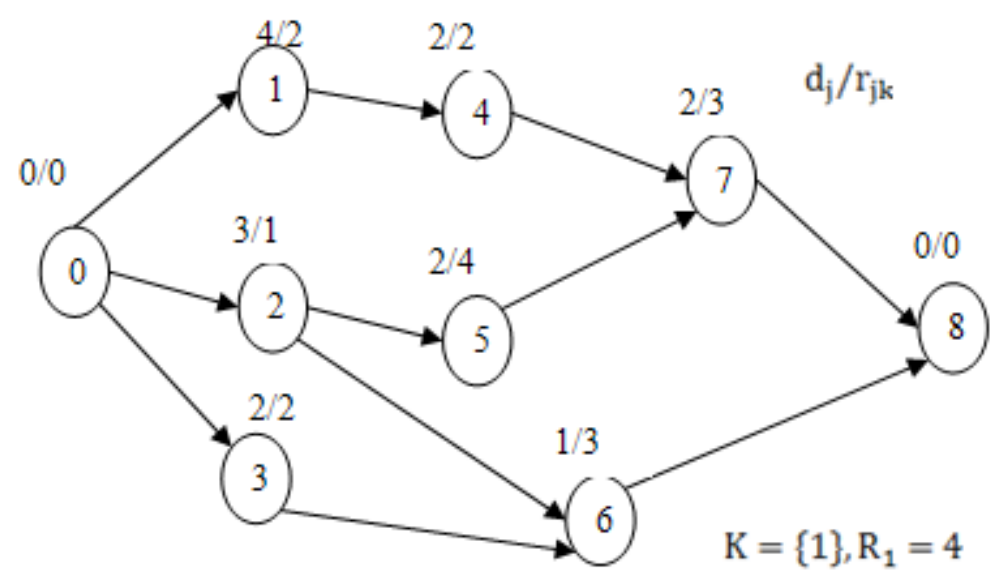

Fig.1. Project network diagram

In the graph shown in Fig.1. If there is a line between the two vertices directly connected to the two vertices, then we call the two vertices adjacent. In the matrix, if two vertices are adjacent, the value is 1 , if not the value is 0 .

\begin{tabular}{cccccccccc} 
& 0 & 1 & 2 & 3 & 4 & 5 & 6 & 7 & 8 \\
0 & 0 & 1 & 1 & 1 & 0 & 0 & 0 & 0 & 0 \\
1 & 0 & 0 & 0 & 0 & 1 & 0 & 0 & 0 & 0 \\
2 & 0 & 0 & 0 & 0 & 0 & 1 & 1 & 0 & 0 \\
3 & 0 & 0 & 0 & 0 & 0 & 0 & 1 & 0 & 0 \\
4 & 0 & 0 & 0 & 0 & 0 & 0 & 0 & 1 & 0 \\
5 & 0 & 0 & 0 & 0 & 0 & 0 & 0 & 1 & 0 \\
6 & 0 & 0 & 0 & 0 & 0 & 0 & 0 & 0 & 1 \\
7 & 0 & 0 & 0 & 0 & 0 & 0 & 0 & 0 & 1 \\
8 & 0 & 0 & 0 & 0 & 0 & 0 & 0 & 0 & 0 \\
\multicolumn{7}{c}{ Table 1 Adjacency matrix M }
\end{tabular}




\section{Improved Quantum Genetic Algorithm}

Quantum genetic algorithm was introducing the state vector of the quantum into genetic encoding, then uses quantum rotation gate to realize the evolution of chromosome, so as to achieve better results than conventional genetic algorithm. Because of the quantum genetic algorithm only considers the global optimization. In this paper, we proposed the inter-cell method by angle encoding, use the idea of $H_{\varepsilon}$ gate to improve the gate update, adopt the dynamic quantum step-adjusting strategy, and introduce new improved quantum genetic algorithm according to the literature [4].

\section{Chromosome encoding}

To represent the prioritized relationship among each individual, we used triangular matrices to represent the ordering of the job of each individual. According to the basic relational matrix $M$ of Table 1, we represent the quantum individual as a triangular matrix consistent with the structure of the relation matrix $M$. The triangular matrix can be represented by a vector containing $(J-1) *(J-2) /$ 2 elements.

$$
q_{i}=\left[\theta_{i 1}, \theta_{i 2}, \ldots ., \theta_{i m}\right]
$$

In the equation (3), $\theta_{i j} \in\left[0, \frac{\pi}{2}\right], i=1,2, \ldots n, j=1,2, \ldots ., m, n$ is the population size, chromosome length $m=(J-1)(J-2) / 2$ which is the number of the triangular matrix elements .

\section{Population initialization}

The initial population generated by the inter-compartment method is evenly distributed in the solution space, which helps to maintain the diversity of the population. In this paper, the inter-cell method is introduced. The core is to divide the quantum bit probability space into $\mathrm{N}$. The initialization process divides the probability space according to Eq. (4), and initializes each sub-population into quantum chromosomes with the same probability. according to the literature [5] $\theta_{k}=\frac{i}{N} \frac{\pi}{2}$

Equation (4) and Fig.2 shows the initial value of the $i$ chromosome in $N$ populations.

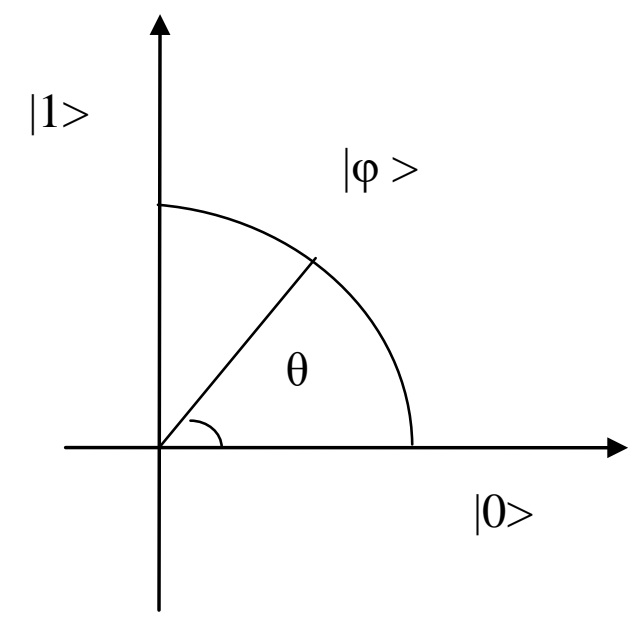

Fig.2. The Angle Representation of Quantum Bit

\section{Quantum gate update}

Quantum gate update operation is the main means of chromosome evolution. Each gene bit of the new coded quantum chromosome is an angle taken on $[0, \pi / 2]$. The effect of the revolving gate on the gene bit is multiplied by the matrix and added to the angle, its form has been simplified, and the 
amount of calculation is greatly reduced. The new rotation gate operation will increase or decrease the chromosomes in the population, and the chromosomes will evolve toward the evolutionary goal. That is, the population converges to the optimal solution while maintaining the diversity of the population. The revolving gate $\mathrm{U}$ can be expressed as:

$\theta^{\prime}=\theta+S(\theta) \Delta \theta$

In the equation (5), $\theta$ 'and $\theta$ are the values of the $j$ gene bit of the chromosome after operation and before operation respectively.

$\Delta \theta$ is the quantum update step size of the value between $0 \sim \pi / 2$, according to the actual situation, you can choose a stable value, and you can also according to the evolution of algebra and other factors to dynamically adjust. Show as Fig.3 .

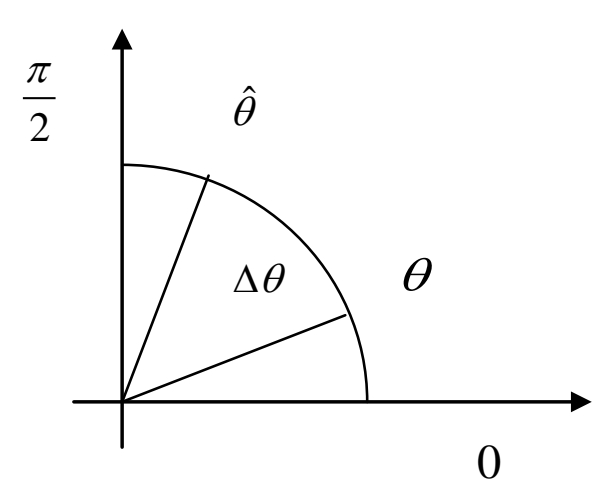

Fig.3. Rotation direction diagram

$\hat{\theta}$ is one of the best individuals in the current search, and $\theta$ is the corresponding gene bit of the individual to be evolved.

$$
S(\Delta \theta)=\left\{\begin{array}{l}
1, \hat{\theta}>\theta \\
-1, \hat{\theta}<\theta \\
0, \hat{\theta}=\theta
\end{array}\right\}
$$

\section{Mutation operation}

In order to ensure the diversity in the process of evolution and avoid the algorithm fall into premature, the quantum mutation operation actually changes the superposition state of the quantum bit state, so that the original tendency to collapse to the state $\mid 1>$ becomes tending to collapse to the state $\mid 0>$; or vice versa. In this paper, according to the literature [6].I will introduce the mutation operation formula.

$\hat{\theta}=\left(\frac{\pi}{2}-\theta\right)$

Mutation probability is the key to determine the mutation operation. In this paper, the value of $p_{m}$ range from 0.01 to 0.1 .

\section{Algorithm steps}

step1: Based on the matrix $M$ to initialize the population: take the initial population $Q(t)$ and determine the population initial value according to Eq. (4)

step2: Quantum collapse: an individual is observed in $Q(t)$ to obtain a set of deterministic solutions $R(t)=\left\{p_{1}{ }^{t}, p_{2}{ }^{t}, \ldots, p_{n}{ }^{t}\right\}$. Where $P_{i}{ }^{t}$ is the observed value of the chromosome $i$ in the $t$ generations, and the form is a binary string of length $m$. The specific operation is that randomly produce a number of $0 \sim \pi / 2$, if it is greater than $\theta_{i j}$, then the observed value of the corresponding gene bit is 1 , otherwise 0 . 
step3: Fitness calculation: the quantum collapse of each group of binary string generated by the function of the variable range of real values, and substituting it into the fitness function. The optimal chromosomes obtained from the past generations is $q_{b}$, current generation optimal chromosome is qs.If $f\left(q_{s}\right)>f\left(q_{b}\right)$ so that $q_{b}=q_{s}$, if not $\boldsymbol{q}_{s}=\boldsymbol{q}_{b}$.

step4: Producing new populations through crossover and mutation operation for the quantum bit population according to Eq. (7).

step5: A new quantum bit populations was generated by the quantum gate according to Eq. (5).

step6: When the iteration algebra $t$ reaches $T$, the evolution is finished and the result is output, otherwise go to step2.

step7: Output current optimal solution.

\section{Test results}

In this paper, we use MATLAB to solve RCPSP under the environment of $2.8 \mathrm{GHz} \mathrm{CPU}$ and 2.0GB memory. The maximum number of iterations Maxgen $=300$ and the population size be 20 . The Mutation probability $\mathrm{Pm}=0.01$, Minimum rotation angle $\theta_{\min }=0.005 \pi$, maximum rotation angle $\theta_{\max }=0.05 \pi$, The algorithm will run 20 times. The three standard problems are simulated by QGA and IQGA respectively.

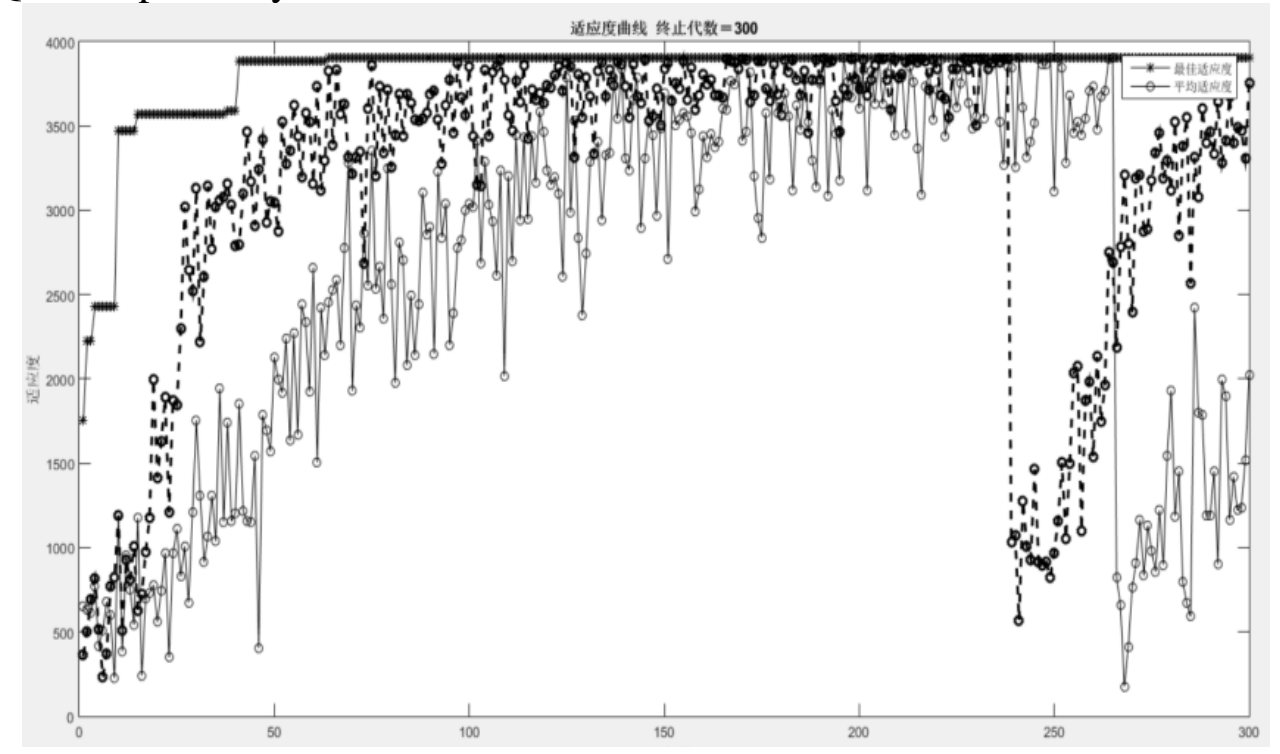

Fig.4. Algorithm fitness curve

In this experiment, we can see from the figure above that the dotted line represents the result calculated by IQGA is closer to the theoretical value, the number of convergence is the most, the number of steps is the least, and the calculation time is shortest. Therefore, in the multi-peak function optimization problem, the performance of IQGA algorithm is better than QGA. In Fig. 4, the optimal solution of IQGA is found near the 110th generation, and the value of the optimal solution decreases in turn. Each chromosome contains more subspaces, so IQGA is more abundant than the traditional QGA and has a wider search range and ability of parallel search.

The single-mode project scheduling problem in the standard problem library PSPLI B is tested. According to the number of jobs included in the project, there are three types of standard problem libraries, 32, 62 and 122 jobs according to the literature [6], all examples of J32 group have been obtained the most of the short period, but the date of J62 and J120 sets has not yet been solved, because the optimal solution PSPUB only provided the best solution so far. The average deviation avg-dev, maximum deviation max-dev, optimal solution and min-gen optimal solution are recorded. The simulation results are shown in Table 2. 


\begin{tabular}{|c|l|l|l|l|l|}
\hline problem & algorithm & avg-dev & max-dev & optimal & min-gen \\
\hline \multirow{3}{*}{ J30 } & QGA & 0.49 & 3.94 & 0.82 & 20.2 \\
\cline { 2 - 6 } & IQGA & 0.32 & 3.22 & 0.93 & 26.7 \\
\hline \multirow{3}{*}{ J60 } & QGA & 10.6 & 15.6 & 0.76 & 19.3 \\
\cline { 2 - 6 } & IQGA & 9.23 & 13.42 & 0.78 & 25.5 \\
\hline \multirow{3}{*}{ J120 } & GQA & 33.9 & 51.9 & 0.18 & 37.5 \\
\cline { 2 - 6 } & IQGA & 27.7 & 44.3 & 0.34 & 45.6 \\
\hline
\end{tabular}

Table 2 Experimental results.

It can be seen from Table 2 that the iterative algorithm IQGA proposed in this paper has better convergence speed and result than other heuristic algorithms. The QGA algorithm takes advantage of the properties of interference and entanglement of the quantum state and the dynamic convergence strategy ,and uses the rotation angle. The convergence rate is the fastest, and the optimal solution is better than the QGA, reflecting the convergence speed and global search capability of IQCA.

\section{Conclusion}

In this paper, we proved that quantum genetic algorithm based on quantum information computing and genetic algorithm can effectively improve the optimization of genetic algorithm and convergence efficiency, to solve large-scale, complex scheduling problem. It plays an especially positive role to solve the problem of resource-constrained project. The experimental results show that the algorithm can improve the optimization efficiency. In the application of quantum genetic algorithm, we think that there are several aspects to be further studied: combining with the traditional algorithm. There are many exact algorithms and heuristic algorithms with high computational efficiency, which can be considered combined with quantum genetic algorithm, proposed quantum hybrid genetic algorithm. Such as the combination of neural network computing theory and quantum genetic algorithm. According to the literature [7].

\section{References}

[1] Gong C Q, Zhang B, Li Y. Resources Scheduling of TT\&C Network Based on Quantum Genetic Algorithm[C].International Conference on Wireless Communications, NETWORKING and Mobile Computing. 2009:1-4.

[2] Ayodele M, Mccall J, Regnier-Coudert O. Probabilistic Model Enhanced Genetic Algorithm for Multi-Mode Resource Constrained Project Scheduling Problem[C]. The Companion Publication of the. 2015:745-746.

[3] Das PP, Khan M. Quantum-Inspired Evolutionary Algorithm to Solve Graph Coloring Problem[C].International Conference on Advances in Computing, Electronics and Electrical Technology - Ceet. 2014:66-70.

[4] Joag P, Mehendale D. Quantum Algorithm for Linear Programming Problems[C]// APS Meeting. APS Meeting Abstracts, 2016.

[5] Deng G, Wei M, Su Q, et al. An effective co-evolutionary quantum genetic algorithm for the no-wait flow shop scheduling problem[J]. Advances in Mechanical Engineering, 2015, 7(12).

[6] Leyman P, Vanhoucke M. A new scheduling technique for the resource-constrained project scheduling problem with discounted cash flows[J]. International Journal of Production Research, 2015, 53(9):2771-2786.

[7] Zamani R. An evolutionary implicit enumeration procedure for solving the resource-constrained project scheduling problem[J]. International Transactions in Operational Research, 2015. 